\title{
Remoção cirúrgica de lipoma em região maxilofacial: relato de caso
}

Surgical removal of lipoma in maxilofacial region: case report

Remoción quirurgíca de lipoma em región maxilofacial: relato de caso

Taysnara Ismaeley de ANDRADE ${ }^{\mathbf{1}}$

Francisco Rikilly de ARAÚJO ${ }^{1}$

Kaiane Tavares PONTES ${ }^{1}$

Arnaldo Pereira BRITO FILHO ${ }^{2}$

Darlan Kelton Ferreira CAVALCANTE ${ }^{3}$

${ }^{1}$ Residência em Cirurgia e Traumatologia Bucomaxilofacial - Hospital Regional do Agreste Dr. Waldemiro Ferreira - Universidade Estadual de Pernambuco (UPE) 55002-970 Indianópolis - PE, Brasil

${ }^{2}$ Especialista em Cirurgia Bucomaxilofacial; Mestre em Odontologia - UFPE - Preceptor da Emergência do Hospital Regional do Agreste pelo Programa de Residência em Cirurgia e Traumatologia Bucomaxilofacial - Hospital Regional do Agreste Dr. Waldemiro Ferreira Universidade Estadual de Pernambuco (UPE) 55002-970 Indianópolis - PE, Brasil

${ }^{3}$ Especialista em Cirurgia e Traumatologia Bucomaxilofacial pela Universidade de Pernambuco (UPE) / Hospital Regional do Agreste (HRA). Mestrando em Perícias Forenses pela Universidade de Pernambuco (UPE) 54730200 Recife-PE, Brasil

\begin{abstract}
Resumo
Introdução: Os lipomas são as lesões tumorais mesenquimais mais encontradas no corpo humano. Sua localização mais comum é em regiões de extremidades e tronco, sendo raras na região maxilofacial. Apresentam-se clinicamente como lesões indolores, de crescimento lento, amolecidas à palpação de base séssil com acometimento em indivíduos de 40 anos, sem predileção por gênero. O tratamento de escolha é a excisão cirúrgica total, apresentando bom prognóstico e baixa taxa de recidiva. Objetivo: relatar um caso de lipoma de grande extensão em região maxilofacial correlacionando com os dados presentes na literatura. Relato de caso: Paciente, gênero masculino, 30 anos de idade, procurou o serviço de cirurgia bucomaxilofacial do Hospital Regional do Agreste Caruaru/PE, com queixas de aumento de volume "embaixo do queixo" com cerca de 1 ano de evolução. Durante anamnese, paciente não relatou nenhuma alergia ou alteração sistêmica. Ao exame físico extra-oral observou-se aumento de volume e assimetria facial, tumefação assintomática de amolecida à palpação em região submandibular esquerda, nodular, de base séssil, com cerca de 4,0 x 3,0 cm de diâmetro. Após diagnóstico, o tratamento escolhido foi biópsia excisional sob anestesia geral. Paciente segue no $2^{\circ}$ ano pós-operatório sem alterações ou recidivas. Conclusão: Conclui-se que os lipomas são lesões tumorais benignas, que devem possuir diagnóstico precoce para instituição do correto tratamento. Apesar de atingirem grandes proporções, possuem bom prognóstico e baixa taxa de recidiva, sendo raras no complexo maxilofacial.
\end{abstract}

Descritores: Lipoma; Cirurgia Bucal; Patologia Clínica.

\section{Abstract}

Introduction: Lipomas are the most found mesenchycal tumor lesions in the human body.Its most common location is in regions of the extremities and trunk, being rare in the maxillofacial region. They present clinically as painless, slow-growing lesions, softened to sessile base palpation with impairment in individuals aged 40 years, With no predilection for gender. The treatment of choice is total surgical excision, presenting good prognosis and low recurrence rate. Objective: To report a case of largeextension lipoma in the Maxillofacial region, correlating with the data present in the literature. Case report: Patient, 30 -year-old male, sought the buccomaxillofacial surgery service of the Regional Hospital of the Agreste Caruaru/PE, With complaints of volume increase "under the chin" with about 1 year of evolution. During anamnesis, the patient reported no allergies or systemic alterations. On physical examination extraoral there was an increase in volume and facial asymmetry, Asymptomatic tumefaction of softened to palpation in the left Submandibular region, Nodular, of Sessile base, with about $4.0 \times 3.0 \mathrm{~cm}$ in diameter.After diagnosis, the treatment chosen was excisional biopsy under general anesthesia. Patient follows in the second postoperative year without alterations or recurrences. Conclusion: It is concluded that lipomas are benign tumor lesions, which should have an early diagnosis for the establishment of the correct treatment.Although they reach large proportions, they have good prognosis and low recurrence rate, being rare in the maxillofacial complex.

Descriptors: Lipoma; Surgery, Oral; Pathology, Clinical.

\section{Resumen}

Introducción: Los lipomas son las lesiones tumorales mensenquimales más encontradas em el cuerpo humano. Su localización más común es em regiones de extremidades y tronco, siendo raras em la región maxiloacial, Se presentan clínicamente como lesiones indoloras, de crecimiento lento, ablandadas a la palpación de base séssil com afectación em individuos de 40 años, sin predilección por gênero. El tratamiento de elección es la escisión quirúrgica total, presentandi buen pronóstico y baja tasa de recidiva. Objetivo: relatar um caso de lipoma de gran extensión em región maxilofacial correlacionando com los datos presentes em la literatura. Relato de caso: Paciente, género masculino, 30 anõs de edad, buscó el servicio de cirurgía bucomaxilofacial del Hospital Regional del Agreste Caruaru/PE, com quejas de aumento de volumen "debajo de la barbilla" com cerca de 1 año de evolucción. Durante la anamnesis, el paciente no reportó ninguna alergia o alteración sistémica. En el examen físico extra-oral se observó um aumento de volumen y assimetria facial, tumefacción assintomática de ablandamiento a la palpacíon em región subamdibular iziquierda, nodular, de base séssil, com cerca de 4,0 × 3,0 cm de diámetro. Después del diagnóstico, el tratamiento elegido fue biopsia excisional bajo anestesia general. El paciente sigue em el $2^{\circ}$ año postoperatorio sin câmbios o recidivas. Conclusión: Se concluye que los lipomas son lesiones tumorales benignas, que deben tener diagnóstico precoz para la instituición del correcto tratamiento. A pesar de alcanzar grandes proporciones, poseen buen pronóstico y baja tasa de recidiva, siendo raras em el complejo maxilofacial.

Descriptores: Lipoma; Cirugía Bucal; Patología Clínica.

INTRODUÇÃO

Os lipomas são lesões tumorais

benignas de origem mesenquimal, considerada uma das mais comuns dos tecidos moles1. Sua localização é frequente em regiões de extremidade e no tronco, sendo relativamente rara na região maxilofacial, com acometimento entre 10 a $20 \%$ dos $\operatorname{casos}^{1,2}$. Sua etiologia é incerta, mas acredita-se que esteja associado a distúrbios endócrinos, trauma local, infecções de origem incerta bem como fatores hereditários. Não apresenta predileção por gênero, sendo as lesões de tronco mais comuns 
no gênero feminino ${ }^{3}$. Sua faixa etária de acometimento é em média indivíduos de 40 anos, sendo raro em crianças ${ }^{4,5}$.

Clinicamente, os lipomas apresentam-se como uma massa nodular, de base séssil ou pedunculada, amolecida à palpação e de superfície lisa, bem circunscrita, assintomática, de tamanhos variados, de coloração amarelada 3,4 . Na cavidade oral, as localizações mais frequentes são língua, assoalho de boca, vestíbulo, palato e mucosa jugal ${ }^{6}$.

Histologicamente, são formados por adipócitos maduros, dispostos em lóbulos caracterizando-se por proliferação lobular de gordura saturada, bem circunscritos, podendo ainda apresentar vasos sanguíneos, tecido conjuntivo fibroso ou músculo. Quando apresentam esses padrões, podem ser classificados em miolipoma, lipoma pleomórfico ou angiolipoma ${ }^{7-9}$.

Como diagnóstico diferencial pode-se citar mucocele, rânula, cisto epidermoide, rabdomiona e fibroma. O tratamento consiste em excisão cirúrgica conversadora, bom prognóstico e recidiva rara ${ }^{9,10}$.

Com base nos dados acima objetiva-se apresentar um relato de caso de lipoma presente em região maxilofacial, correlacionando-os com a literatura vigente.

\section{CASO CLÍNICO}

Paciente do gênero masculino, leucoderma, 30 anos, procurou o serviço de Cirurgia Bucomaxilofacial do Hospital Regional do Agreste- HRA com queixas de aumento de volume em região submandibular esquerda, sem sintomatologia dolorosa, com cerca de 1 ano de evolução (Figura 1).

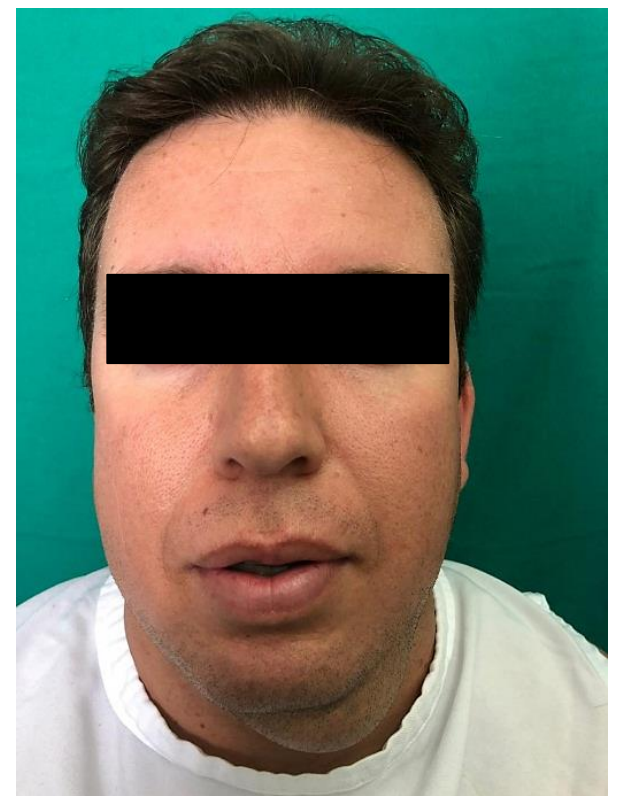

Figura 1: Aspecto facial frontal.
Durante anamnese não foi relatada nenhuma alteração sistêmica ou alergias. Ao físico extraoral foi observado aumento de volume e assimetria facial tumefação assintomática de consistência amolecida à palpação em região submandibular esquerda, nodular, de base séssil, sem ulceração ou qualquer outra alteração, com cerca de 4,0 x 3,0 $\mathrm{cm}$ em seu maior diâmetro, de superfície lisa (Figuras 2 e 3).

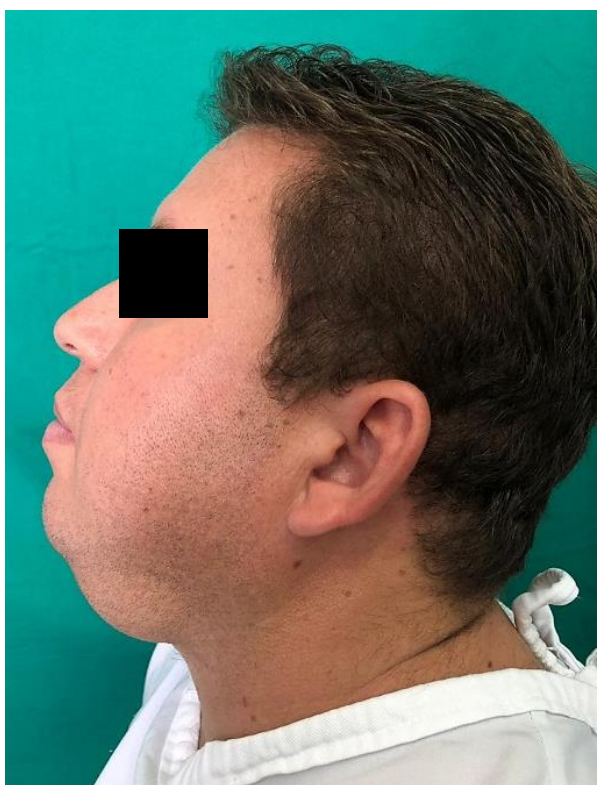

Figura 2: Aspecto facial lateral esquerdo.

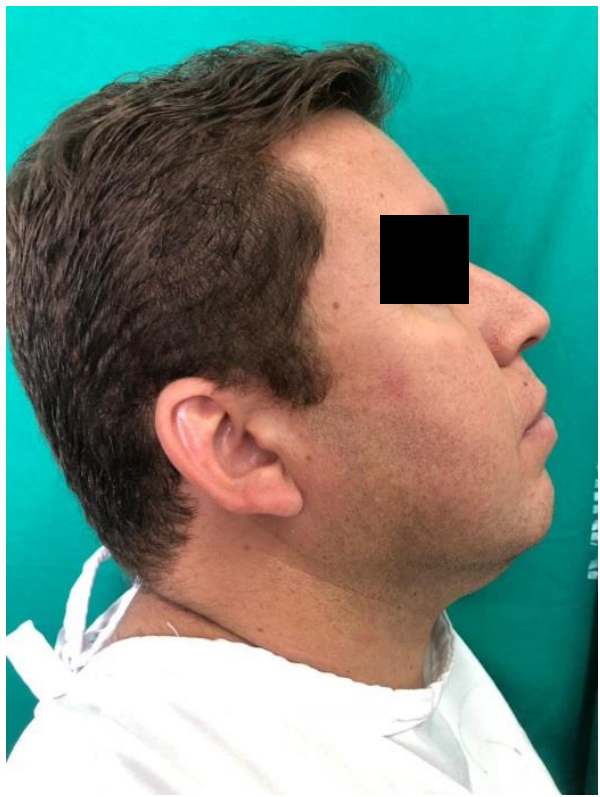

Figura 3: Aspecto facial lateral direito.

Após realização de Punção Aspirativa por Agulha Fina (PAAF), e diagnóstico sugestivo de lipoma, o tratamento escolhido foi a remoção total do tumor. Foi induzida anestesia geral e intubação nasotraqueal, e realizada uma incisão (Figura 4) em região submandibular esquerda, seguida de divulsão e exérese do tumor (Figura 5). A síntese por planos foi realizada com fio Vicryl 4-0, e espécime cirúrgico encaminhado 
para exame histopatológico (Figura 6). Após 8o dia pós-operatório, paciente retornou para reavaliação e portando laudo histopatológico compatível com lipoma. Paciente segue no $2^{\circ}$ ano pós-operatório sem recidivas ou queixas.

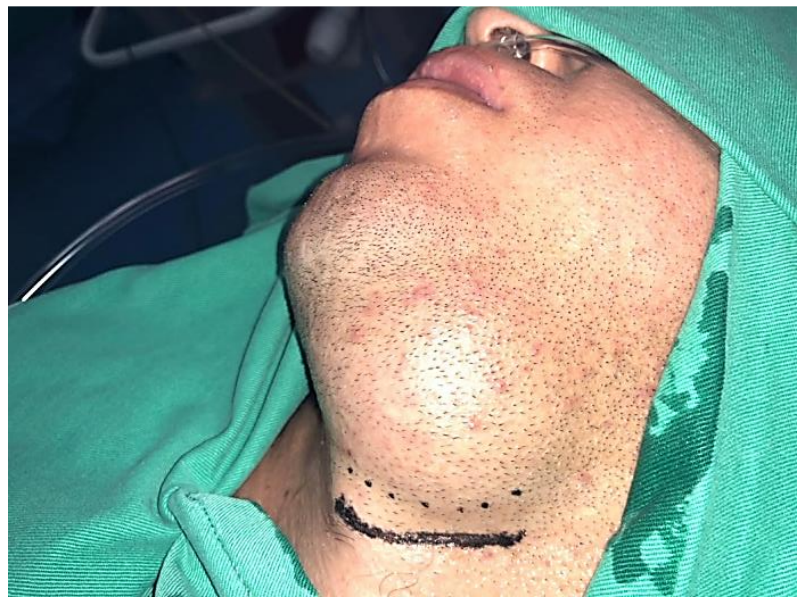

Figura 4: Demarcação da incisão.

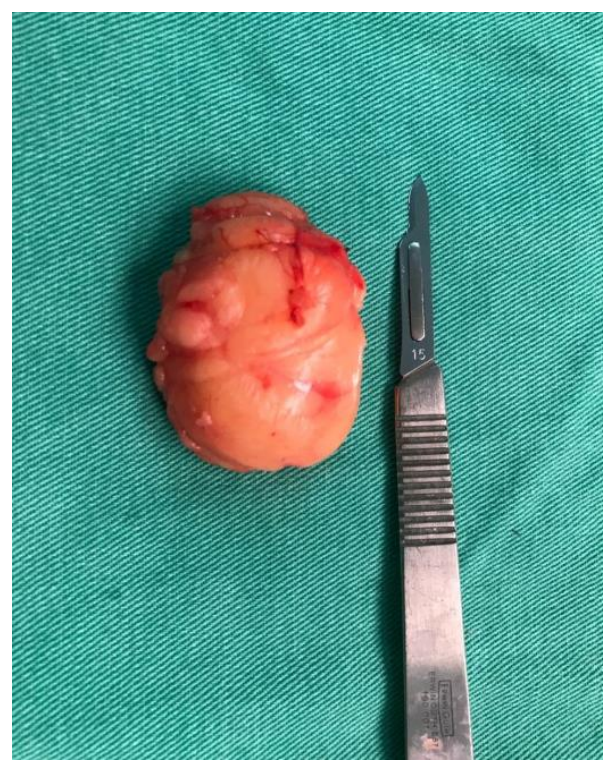

Figura 5: Espécime cirúrgico após exérese total.

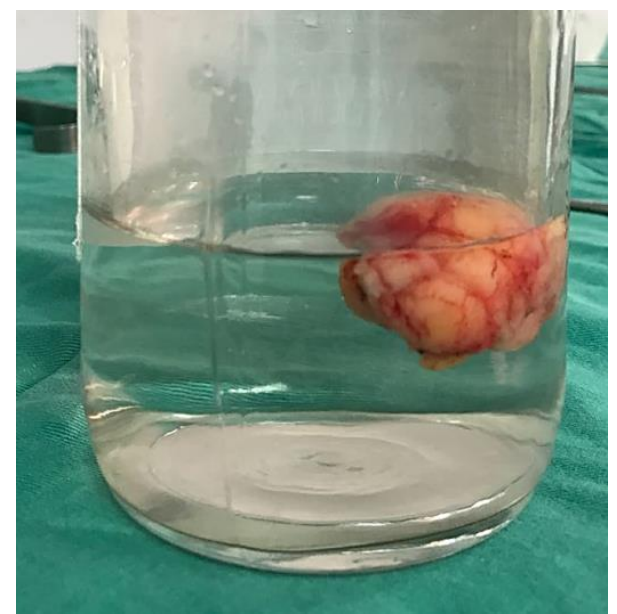

Figura 6: Aspecto "flutuante" característico de lipomas.

DISCUSSÃO

Os lipomas são tumores benignos, de origem mesenquimal advindos de tecido adiposo, sendo relativamente incomuns na região maxilofacial, incluindo a cavidade oral ${ }^{10}$. Não apresentam predileção por gênero, acometendo uma faixa etária média entre 30 e 40 anos $^{11}$. O presente caso apresenta-se em indivíduo de 30 anos de idade, gênero masculino, com lipoma de grande extensão em região submandibular esquerda.

Apesar de possuir etiologia incerta, muitos autores acreditam que essas lesões tumorais estão relacionadas a distúrbios endócrinos, fatores hereditários ou traumas pregressos, além de possíveis infecções locais, porém ainda nenhum fator parece estar diretamente relacionado ao seu aparecimento ${ }^{12}$. No caso relato, o paciente negou qualquer desses fatores etiológicos bem como não foram observadas alterações endócrinas.

Sua localização é mais comum em regiões de tronco e extremidades, sendo relativamente raro na região maxilofacial ${ }^{8,9}$. Quando acometido, os lipomas nessa região, apresentam-se em maior frequência na cavidade oral, em região de mucosa jugal. Lipomas em espaços fasciais são raros, em especial submandibular. $O$ caso relatado apresenta lesão isolada em região submandibular esquerda, que é um espaço fascial primário ${ }^{10,11}$.

No que se refere às características clínicas e histológicas, o caso apresentou quadro típico conforme a literatura: lesão única, de crescimento lento e assintomático, amolecida à palpação, base séssil, apresentando histologicamente adipócitos maduros, bem circunscritos e delimitadas por uma cápsula de tecido fibroso, com hemácias em seu interior ${ }^{6-8}$.

Os exames por imagem são reportados como adjuvantes no diagnóstico dessas lesões, sendo os mais utilizados a tomografia computadorizada, e a ultrassonografia. Também é relatado a PAAF, que se trata de uma biópsia guiada por agulha fina para avaliação histológica prévia, auxiliar na determinação do tratamento a ser escolhido ${ }^{2-4}$. Foi solicitada PAAF e ultrassonografia para avaliação e diagnóstico diferencial, o qual apresentou imagem nodular com textura adiposa, de contornos regulares e definidos, sem vascularização detectável, localizada em tecido subcutâneo com tecido fibroadiposo maduro e hemácias.

Após diagnóstico, o tratamento escolhido foi a exérese total da lesão, através de biópsia excisional, sob anestesia geral, conforme relatado pela literatura ${ }^{1-3,13}$. Realizada a exérese e espécime acomodado para encaminhamento ao histopatológico, foi possível observar lesão flutuante, como relatado na literatura. 


\section{CONSIDERAÇÕES FINAIS}

Os lipomas são lesões raras no complexo maxilofacial, sendo assim importante um diagnóstico precoce, pois apesar de tratarem-se de neoplasia benignas, podem atingir grandes proporções e acarretar e alterações para os indivíduos acometidos. Os exames de imagem são importantes para o diagnóstico e o tratamento cirúrgico é a exérese total da lesão, apresentam um bom prognóstico e baixa taxa de recidiva.

\section{REFERÊNCIAS}

1. Tenório JR, Paiva KM, Nogueira PTBC, Silva EDO. Exérese de extenso lipoma em região submandibular: Relato de caso. Rev cir traumatol buco-maxilo-fac. 2013;13(3):43-50.

2. Costa DFN, Inaoka SD, Silveira KG, Souza NL, Santos LAM. Tratamento cirúrgico de lipoma: Relato de dois casos. Rev cir traumatol bucomaxilo-fac. 2017;17(3):25-28.

3. Santos RR, Souza DO. Tratamento de lipoma em mucosa jugal: relato de caso. Rev Ciênc Méd Biol. 2017;16(2):248-50.

4. Marques DO, Silva GA, Brigantini LC, Araújo C, Araújo CSA, Gonçalves KJ et al. Lipoma em mucosa jugal: Relato de caso. Rev Uningá, 2014;20(3):40-42.

5. Resende R, Meirelles M, Varella R. Remoção cirúrgica de grande proporção: Relato de caso. Rev cir traumatol buco-maxilo-fac. 2013; 13(2):37-42.

6. Santos LAM, Barbalho JCM, Costa DFM, Silva CCG, Pereira VBS, Vasconcelos BEC. Lipoma intra-oral: Relato de caso. Rev cir traumatol buco-maxilo-fac. 2014:14(3):39-44.

7. Dehghani N, Razmara F, Padeganeh T, Mahmoudi X. Oral lipoma: Case report and review of literature. Clin Case Rep. 2019;7: 809-15.

8. Babu SS, Sunil S, Pratap N, Thomas E. Intraosseous lipoma of the maxillary tuberosity: A rare entity with diagnostic challenge. J Can Res Ther 2019;15:173-76.

9. Silva ALG, Correa MS, Magalhães AM, Freitas CAF, Silva LFG, Pancini ID. Lipoma de localização rara na cavidade oral: relato de caso. Rev. Bras Cir Cabeça Pescoço. 2016;45(2):56-8.

10. Peres PI, Barros RMG, Cieslak SR, Saldanha KDF, Costa DC, Jardim ECG. Lipoma intrabucal: um caso atípico. Salusvita. 2015;34(2):317-25.

11. Melo DF, Manica MZ, Helene Júnior A. Lipomas gigantes: série de 14 casos. Rev Bras Cir Plást. 2015;30(1):33-7.

12. Chia GY, Rovaris DA, Fontana R. Lipoma gigante de coxim adiposo bucal: relato de caso e revisão de literatura. Rev Bras Cir Plást. 2016;31(1):112-17.
13. Castro TF, Biss SP, Figueira JA, Tomo $S$, Biasoli ER, Bernabé DG, Crivelini MM, Miyahara Gl. Ultrassonografia como ferramenta no diagnóstico do lipoma em região maxilofacial: relato de caso. Arch Health Invest. 2018;7 (Spec Iss 3):467.

\section{CONFLITO DE INTERESSES}

Os autores declaram não haver conflitos de interesse

\section{AUTOR PARA CORRESPONDÊNCIA}

Darlan Kelton Ferreira Cavalcante

Programa de Pós-Graduação em Perícias Forenses Universidade de Pernambuco (UPE)

54730200 Recife-PE, Brasil

Email: darlankelton@hotmail.com

Submetido em 30/06/2019

Aceito em 23/10/2020 